\title{
LES USAGES ORGANISATIONNELS DES ESPACES GÉOGRAPHIQUES
}

\author{
Matthieu MANDARD \\ Maître de conférences en sciences de gestion \\ Centre de Recherche en Économie et Management (CREM - UMR \\ CNRS 6211) \\ Université Rennes 1 \\ 11 rue Jean Macé - CS 70803 - 35708 RENNES Cedex 7 - FRANCE \\ E-mail : matthieu.mandard@univ-rennes1.fr
}


Le rôle des espaces géographiques dans les activités des organisations est peu examiné dans la littérature. L'objectif de cet article est de pallier ce problème en montrant que les usages organisationnels des espaces géographiques sont en nombre limitéd'ordre écologique, économique, esthétique ou symbolique - et que chacun présente des caractéristiques spécifiques. Il est aussi de souligner le fait que ces usages ne s'imposent pas d'eux-mêmes : ils sont le produit de conflits d'intérêts. Cet article offre ainsi une synthèse d'un sujet d'actualité qui emporte une série d'implications pratiques et des pistes de recherche à venir.

Mots-clefs : écologie, économie, espace géographique, esthétique, symbolique

The role of geographic spaces in organizations' activities has received little attention in the literature. The objective of this paper is to fill this gap by showing that the organizational uses of geographical spaces are in limited number - ecologic, economic, aesthetic, or symbolic - and that each use displays specific characteristics. It is also to highlight the fact that these uses are not self-imposed: they are the product of conflicts of interest. This paper thus offers a synthesis of a hot topic that carries a series of practical implications as well as avenues for future research.

Keywords: aesthetic, ecology, economy, geographic space, symbolic

El papel de los espacios geográficos en las actividades de las organizaciones está poco estudiado en la literatura. El objetivo de este artículo es superar este problema mostrando que los usos organizativos de los espacios geográficos son limitados en número ecológicos, económicos, estéticos o simbólicos - y que cada uno tiene características especificas. También es para enfatizar el hecho de que estos usos no se imponen : son el producto de conflictos de intereses. Este artículo ofrece una sintesis de un tema actual que conlleva una serie de implicaciones prácticas y pistas para futuras investigaciones.

Palabras claves : ecología, economía, espacio geográfico, estética, simbólica 


\section{Introduction}

Le rôle des espaces géographiques dans les activités des organisations est peu examiné dans la littérature académique. La plupart du temps, lorsque la géographie est évoquée, c'est uniquement en considérant la distance entre une organisation et ses partiesprenantes - avec les travaux sur la proximité géographique et la proximité organisée au cœur desquels se trouve le concept de territoire (Zimmermann, 2008) - ou en examinant les pays dans lesquels une organisation opère - avec les travaux sur les stratégies d'internationalisation (Krugman, 1991) -, ignorant de la sorte les propriétés des espaces au sein desquels les organisations sont insérées. Ceci est problématique, puisque les organisations ne déploient pas leurs activités dans le vide : elles sont encastrées dans des contextes géographiques qui tout à la fois permettent et contraignent ces activités.

L'objectif de cet article est de pallier ce problème en montrant que les usages organisationnels des espaces géographiques sont en nombre limité - d'ordre écologique, économique, esthétique ou symbolique - et que chaque usage présente des caractéristiques qui lui sont spécifiques. Il est aussi de souligner le fait que ces usages ne s'imposent pas d'eux-mêmes : ils sont le produit de conflits d'intérêts. La définition de la nature des usages des espaces géographiques comme l'obtention du droit de faire usage de ces espaces font en effet fréquemment l'objet de luttes entre parties-prenantes (Briec, 2015).

L'intérêt d'un tel travail est double. D'un point de vue managérial, il offre un panorama d'un sujet particulièrement d'actualité qui emporte une série d'implications pratiques. D'un point de vue scientifique, il permet de délimiter ce sujet en tant que champ d'investigation scientifique et d'identifier les perspectives de recherche à venir.

L'article est structuré comme suit. Nous proposons tout d'abord une typologie qui met en évidence les quatre principaux usages des espaces géographiques. Nous poursuivons par une présentation des caractéristiques clefs de chacun de ces quatre usages et nous fournissons des exemples. Nous mettons ensuite en évidence les conflits qui peuvent exister dans la définition des usages des espaces géographiques comme dans l'obtention du droit à user de ces espaces. Nous concluons en recensant les implications de ce travail. 


\section{Les usages organisationnels des espaces géographiques : une proposition de typologie}

L'examen des usages des espaces géographiques recensés dans la littérature permet de faire émerger quatre principaux types d'usages: un usage économique, un usage écologique, un usage esthétique et un usage symbolique (Tableau). Il apparaît en effet que tout usage des espaces géographiques relève de l'un ou l'autre de ces types, ou de leur combinaison. L'objectif de cette section est de proposer une typologie qui montre que ces quatre principaux usages se différencient selon deux dimensions essentielles : l'intérêt porté à l'espace géographique et la manière dont cet espace est employé.

Nous tenons à souligner qu'il s'agit là d'une proposition de théorisation, qui tente de rendre compte des faits observés. Un tel travail de théorisation ne peut trouver sa justification que dans la qualité de l'adéquation qu'il entretient avec le réel (principe de vérisimilitude). En d'autres termes, il peut être jugé comme acceptable s'il comprend bien tous les usages organisationnels des espaces géographiques connus - ce qui nous semble être le cas.

Tableau - Les usages organisationnels des espaces géographiques : une proposition de typologie

\begin{tabular}{cc}
\hline \multicolumn{1}{c}{$\begin{array}{c}\text { Intérêt porté à l'espace } \\
\text { géographique }\end{array}$} \\
\hline $\begin{array}{c}\text { Intérêt } \\
\text { intrinsèque } \\
\text { Intérêt } \\
\text { extrinsèque }\end{array}$ \\
\hline Écologique & Économique \\
\hline Esthétique & Symbolique \\
\hline
\end{tabular}




\subsection{L'intérêt porté à l'espace géographique}

Depuis les années 1960, les psychologues distinguent communément deux types de motivations à l'origine des actions des individus (Ryan et Deci, 2000). Une motivation intrinsèque est définie comme un intérêt inhérent à la tâche à accomplir, alors qu'une motivation extrinsèque renvoie usuellement à un intérêt dans des aboutissements qui sont séparés de la tâche à accomplir. Cette distinction est depuis longtemps utilisée dans l'étude des comportements au travail afin de décrire et d'expliquer la motivation des employés (Ambrose et Kulik, 1999).

Dans cet article, nous proposons d'adopter cette dichotomie afin d'inventorier les usages organisationnels des espaces géographiques. L'usage d'un espace peut tout d'abord être qualifié d'intrinsèquement motivé lorsqu'il existe un intérêt inhérent aux propriétés de cet espace. Tel est le cas lorsque des espaces sont employés à des fins écologiques ou esthétiques : il existe alors un intérêt spécifiquement porté à ces espaces, puisqu'il s'agit respectivement d'exploiter les ressources que ces espaces sont en mesure de fournir (e.g., forêts gérées durablement, sites de pêche raisonnée, zones d'agriculture responsable), ou d'admirer la beauté de ces espaces en raison de leurs propriétés intrinsèques (e.g., volcans d'Auvergne, lac Baïkal, Grand Canyon). A contrario, l'usage d'un espace peut être qualifié d'extrinsèquement motivé lorsqu'il existe un intérêt dans des aboutissements qui ne relèvent pas des propriétés de cet espace à proprement parler. Tel est le cas lorsque des espaces sont employés à des fins économiques ou symboliques. Il n'existe pas d'intérêt spécifiquement porté aux propriétés de ces espaces, puisqu'il s'agit respectivement d'en exploiter le sol indépendamment de ce qu'ils sont véritablement en mesure d'offrir, jusqu'à parfois en détruire totalement l'écosystème (e.g., zone de surpêche, usine polluante, complexe commercial géant $)^{1}$, ou d'attribuer à ces espaces une signification qui dépasse leurs propriétés intrinsèques (e.g., montagne sacrée, terre sainte, frontière fluviale).

1 Nous avons conscience d'attribuer un sens restrictif à la notion d'usage économique des espaces géographiques, que nous définissons comme un usage qui ne respecte pas les propriétés intrinsèques de cet espace, et que nous opposons à un usage qualifié d'écologique, que nous définissons comme un usage qui en respecte les propriétés intrinsèques. Nous précisons nos propos dans la section 2. 
Bien entendu, motivations intrinsèques et extrinsèques ne sont pas mutuellement exclusives, dans la mesure où elles peuvent toutes deux être à l'origine d'une action (Ryan et Deci, 2000). Ainsi, un individu pourra être à la fois motivé par le contenu de son travail (motivation intrinsèque) et par la rémunération ou par la réputation que ce travail lui permet d'obtenir (motivation extrinsèque). De la même manière, un même espace géographique peut tout à la fois être exploité durablement (usage écologique motivé par un intérêt intrinsèque) et se voir attribuer une signification particulière (usage symbolique motivé par un intérêt extrinsèque). C'est par exemple le cas de l'usage de la forêt Amazonienne par les amérindiens, qui offre à cette population des ressources vivrières en même temps qu'elle revêt pour eux une dimension sacrée.

\subsection{La manière dont l'espace géographique est employé}

Dans cet article, nous proposons également d'adopter une distinction populaire entre rationalité instrumentale et substantive proposée par Max Weber (1921). Celui-ci postule deux principales fonctions aux actions individuelles : une rationalité instrumentale est à l'œuvre lorsque des actions sont vues comme des moyens, alors qu'une rationalité substantive est présente lorsque des actions sont vues comme des finalités. Dans le premier cas, les actions réalisées sont considérées comme des outils qui permettent d'atteindre un objectif particulier. Dans le second, les actions entreprises sont déterminées par un système de valeur dont le respect constitue un objectif en lui-même. Ainsi, lorsqu'une entreprise adopte des pratiques écologiquement vertueuses, on parlera de rationalité substantive si ces pratiques sont guidées par un souci de respect de l'environnement, tandis que l'on parlera de rationalité instrumentale si ces pratiques sont guidées par une volonté de bénéficier d'une réputation positive auprès de ses parties-prenantes.

De manière analogue, il est possible de distinguer deux types de rationalité à l'origine de l'usage des espaces géographiques. L'usage d'un espace peut être qualifié d'instrumentalement rationnel lorsque cet espace constitue un moyen d'approvisionnement en ressources. Il s'agit typiquement des usages écologiques et économiques, où les espaces géographiques sont vus comme des vecteurs d'approvisionnement en ressources diverses au travers de l'exploitation des sols. A contrario, l'usage d'un espace peut être 
qualifié de substantivement rationnel lorsque cet espace représente une finalité en lui-même. Il s'agit ici des usages esthétiques et symboliques, où les espaces géographiques sont vus en eux-mêmes comme des endroits de manifestation de beauté ou d'idées.

De même qu'en matière de motivation, les deux types de rationalité ne sont pas mutuellement exclusives, dans la mesure où elles peuvent toutes deux être à l'origine d'une action. Ainsi, une entreprise peut adopter des pratiques écologiquement vertueuses à la fois par respect de l'environnement (rationalité substantive) et par souci de bénéficier d'une réputation positive auprès des partiesprenantes (rationalité instrumentale). De la même manière, un même espace géographique peut tout à la fois être employé comme moyen d'approvisionnement en ressources (usage écologique selon une rationalité substantive), et être considéré comme beau (usage esthétique selon une rationalité instrumentale). C'est le cas de la plupart des espaces naturels protégés, maritimes ou terrestres.

\section{Les caractéristiques des usages organisationnels des espaces géographiques}

Nous présentons ici les caractéristiques des quatre principaux usages organisationnels des espaces géographiques précédemment mis en évidence, et nous illustrons ces usages par des exemples.

\subsection{L'usage écologique}

Le premier usage organisationnel des espaces géographiques que notre typologie permet de mettre en évidence est d'ordre écologique. Deux éléments caractérisent ce type d'usage au sens où nous l'entendons ici. D'une part, l'espace géographique est perçu comme un écosystème, c'est-à-dire, en son sens premier, comme un ensemble composé d'un milieu naturel et des êtres vivants qui y vivent. D'autre part, les grands équilibres de cet écosystème ont vocation à être respectés, les organisations ne prélevant que les ressources que cet espace est susceptible de leur offrir de manière durable (Boutaud et Gondran, 2009).

Ici donc, l'usage écologique des espaces géographiques est gouverné par une rationalité instrumentale, dans la mesure où l'espace 
géographique constitue un moyen pour obtenir des ressources. Cet usage est en outre intrinsèquement motivé, dans la mesure où l'espace géographique est employé - exploité - en fonction de ses propriétés particulières.

Il s'agit typiquement d'un usage des espaces géographiques mis en œuvre par des organisations telles que celles qui opèrent dans l'exploitation forestière durable, la pêche durable, l'agriculture responsable ou, plus généralement, par toute organisation qui respecte les propriétés naturelles des espaces qu'elle occupe. C'est afin de tendre vers un tel usage écologique que le leader français de la fabrication d'enveloppes, l'entreprise Pocheco, a par exemple mis en place de nombreuses initiatives telles que des toitures végétalisées pour l'isolation, des capteurs photosensibles pour ajuster la luminosité de l'éclairage de ses installations, ou encore, une bambouseraie pour le traitement des eaux usées (Cardinal, 2013). Toute la difficulté de ce type de démarche consiste à identifier précisément les propriétés de l'espace exploité afin d'en respecter les grands équilibres, au risque sinon de basculer dans un usage économique - nous présentons les termes de ce débat dans la section suivante.

Rencontrant un intérêt croissant dans la littérature en sciences de gestion (Berchicci et King, 2007), cet usage des espaces géographiques se développe en pratique en raison de l'essor que connaît la question du développement durable depuis les années 1980 et la parution du rapport Brundtland (1987) - l'écologie en constituant l'un des piliers avec la question économique et la question sociale. L'essor du développement durable a en effet donné lieu à l'apparition de diverses initiatives telles que le création en 1995 du Conseil mondial des affaires pour le développement durable, association de près de 200 grandes entreprises internationales engagées en faveur du développement durable, ou telles que le Pacte mondial, Charte de l'ONU adoptée en 2000 et visant entre autres à promouvoir une attitude respectueuse de l'environnement de la part des entreprises (Aggeri et Godard, 2009) ${ }^{2}$. Cet essor a également donné lieu à l'émergence des normes ISO 14000, qui encouragent les entreprises à maîtriser les impacts environnementaux de leurs activités - c'est ce que l'on appelle le management environnemental - et qui connaissent une diffusion internationale. Sur ce sujet, on peut enfin noter

2 Un bémol doit cependant être apporté à ce tableau : si les intentions affichées par les participants à ces initiatives sont louables, nombreux sont les observateurs qui y voient là des pratiques de greenwashing... 
l'adoption en 2017 en France de la loi relative au devoir de vigilance des sociétés mères et des sociétés donneuses d'ordre qui, entre autres objectifs, vise non-seulement à prévenir les incidences environnementales des activités des grandes organisations, mais également celles des activités de leurs filiales, de leurs sous-traitants ou de leurs fournisseurs, et ce sur le territoire français comme à l'étranger.

\subsection{L'usage économique}

Le second usage organisationnel des espaces géographiques que notre typologie permet de mettre en évidence est d'ordre économique. L'espace est ici vu comme un inépuisable pourvoyeur de ressources, sans préoccupations pour la durabilité des pratiques mises en œuvre. Il s'agit là d'une vision des espaces géographiques qui précède l'approche écologique; elle est notablement portée par les Lumières qui voyaient le progrès comme la capacité de l'homme à dompter et à asservir la nature à son profit (Boutaud et Gondran, 2009).

De la même manière que pour l'usage écologique, l'espace géographique constitue donc un moyen pour se procurer des ressources (avec donc à l'œuvre une rationalité instrumentale). Ici cependant, cet usage est extrinsèquement motivé, dans la mesure où l'espace géographique n'est pas employé selon ses propriétés naturelles, mais afin d'exploiter les sols indépendamment de leur capacité à fournir durablement des ressources.

Des exemples archétypiques de ce genre d'usage organisationnel sont les sites de stockage de déchets radioactifs, l'agriculture extensive et, plus généralement, toute industrie polluante, comme cela est le cas de l'usine d'alumine du sud-est de la France qui se mit à déverser des millions de tonnes de boues rouges hautement toxiques dans la méditerranée à partir des années 1960 (Loison et Pezet, 2006). C'est également le cas des projets contestés de mégacentres commerciaux qui fleurissent en France à l'heure actuelle. Et c'est aussi le cas des entreprises qui extraient le gaz de schiste, activité qui engendre une pollution de l'environnement considérable.

Mais, plus précisément, quelle est l'exacte démarcation entre un usage écologique et un usage économique d'un espace géographique ? Ce questionnement a récemment été engagé à 
l'échelle de la planète dans une tentative de délimiter les limites planétaires (planetary boundaries), c'est-à-dire d'identifier un espace sûr permettant la réalisation des activités humaines dans le respect du système Terrestre. Cet espace sûr est circonscrit par neuf paramètres (et leurs seuils associés) : le changement climatique, le taux de perte de biodiversité, l'interférence avec les cycles de l'azote et du phosphore, la diminution de la couche d'ozone stratosphérique, l'acidification des océans, la consommation d'eau douce, la modification de l'usage des sols, la pollution chimique, et la concentration atmosphérique en aérosols (Rockström et al., 2009) ${ }^{3}$.

Ce débat a récemment été importé au niveau des organisations par Gail Whiteman et ses collègues dans une tentative de mettre en évidence les fondations écologiques d'une soutenabilité organisationnelle (2013). Prenant le cas d'activités conduites par l'entreprise Unilever, ils ont montré la mesure dans laquelle ces activités pouvaient localement affecter trois limites planétaires - le changement climatique, le taux de perte de biodiversité et la modification de l'usage des sols -, et ainsi être classifiées comme écologiques ou purement économiques en fonction des seuils qu'elles atteignent. Cette recherche exploratoire a depuis été largement reprise par des travaux ultérieurs, qui tentent d'identifier les seuils de soutenabilité des activités organisationnelles à l'échelle des organisations elles-mêmes (Pogutz et Martín-Tapia, 2017). L'objectif est de pouvoir à l'avenir identifier précisément la démarcation entre usage économique et usage écologique d'un espace géographique par une organisation. En complément des politiques étatiques déployées à une échelle globale, il apparaît en effet dorénavant nécessaire que les organisations s'emparent des questions environnementales à un niveau local pour que la cause écologique progresse (Green et al., 2017).

\subsection{L'usage esthétique}

Troisième rôle considéré, l'usage esthétique des espaces géographiques prend son essor à la Renaissance avec l'apparition de la notion de paysage, qui caractérise précisément un espace doté de qualités esthétiques. Cet intérêt se développe peu à peu au cours des

\footnotetext{
3 D'autres indices de mesure de la soutenabilité environnementale des activités humaines existent, tels que l'empreinte écologique, l'indice de vulnérabilité environnementale ou l'indice de bien-être durable, mais celui de limites planétaires s'avère offrir une plus grande validité et une plus grande fiabilité (Whiteman, Walker et Perego, 2013).
} 
siècles qui suivent dans les sociétés occidentales. La nature n'est plus seulement vue comme une pourvoyeuse de ressources : elle est en elle-même un spectacle (Paquot, 2016).

Cet usage est gouverné par une rationalité substantive, dans la mesure où l'espace géographique constitue une finalité: il représente en lui-même une manifestation de beauté. Il est en outre motivé intrinsèquement, dans la mesure où l'espace géographique est employé en fonction de ses propriétés particulières. La beauté est en effet associée à des caractéristiques paysagères spécifiques, même si cette association peut varier en fonction des cultures - des exemples populaires : les volcans d'Auvergne, le lac Baïkal, le Grand Canyon.

De nos jours, bien que particulièrement négligé, de nombreuses organisations telles que des pôles de compétitivité (e.g., le pôle Bretagne Atlantique près de Brest), des campus universitaires (e.g., le campus de l'INSEAD à Fontainebleau), ou des entreprises touristiques (e.g., les restaurants de bord de mer) s'établissent volontairement dans des endroits jugés esthétiques. De telles localisations leurs permettent en effet d'apparaitre attractives et ce, pour les employés comme pour les consommateurs.

Du point de vue de la gestion des ressources humaines, l'esthétique des sites d'implantation des entreprises influence positivement le recrutement, la motivation et la fidélisation des employés. C'est l'argument sur lequel s'appuient par exemple de petites entreprises innovantes de l'ouest de l'Irlande pour attirer leurs employés: elles ne disposent pas des mêmes moyens que leurs concurrents de grande taille en matière de rémunération ou d'évolutions de carrière, mais elles peuvent se prévaloir d'un cadre naturel exceptionnel (White, 2010). D'un point de vue marketing également, si les travaux en matière d'esthétique ont avant tout porté sur le design des produits, l'esthétique des espaces géographiques occupés par les organisations est aussi d'importance, puisqu'il est susceptible d'influencer positivement la venue de clients et l'expérience des consommateurs : c'est bien entendu l'argument clef de l'industrie du tourisme (Kirillova, Fu, Lehto et Cai, 2014). À l'inverse, il convient de noter que les sites considérés comme inesthétiques freinent souvent la venue d'employés et de clients, et donc celle des organisations. 


\subsection{L'usage symbolique}

Dernier usage des espaces géographiques que nous mettons en évidence, l'usage symbolique a été particulièrement étudié en sociologie. Il s'incarne dans la notion de lieu, qui est un endroit caractérisé par les idées qu'il représente. Un tel usage de l'espace n'est pas nouveau, puisqu'il semble qu'il y ait toujours eu des idées associées à des espaces: des lieux profanes et des lieux sacrés, des lieux publics et des lieux privés, ou encore, des lieux de travail et des lieux de repos (Gieryn, 2000).

Ici, de la même manière que pour l'usage esthétique, l'usage est gouverné par une rationalité substantive, dans la mesure où l'espace géographique constitue une finalité : il constitue en lui-même une manifestation d'idées. En revanche, l'usage est ici extrinsèquement motivé, car l'espace géographique n'est pas employé selon ses propriétés naturelles: des idées peuvent en effet être associées à n'importe quel espace géographique - des exemples classiques : montagne sacrée, terre sainte, frontière fluviale.

$\mathrm{Au}$ niveau des organisations, la théorie institutionnelle a largement montré que celles-ci doivent afficher certaines caractéristiques et symboles si elles veulent être perçues comme légitimes (DiMaggio et Powell, 1983). C'est pourquoi, entre autres raisons, de nombreuses start-up s'établissent dans la Silicon Valley afin d'être perçues comme innovantes, des entreprises du secteur du luxe s'installent - ou installent leur magasin phare : leur flagship - à Paris afin d'être perçues comme prestigieuses, et la City de Londres attire les compagnies financières car elle offre un signal de qualité pour ce genre d'activité (Zimmerman et Zeitz, 2002). Une recherche montre ainsi que, jusqu'à la nationalisation de l'électricité en 1946, les anciennes compagnies françaises d'électricité installaient traditionnellement leurs sièges sociaux en plein cœur de Paris afin d'asseoir leur image de marque (Chaumier, 2010). À l'inverse, des endroits tels que les pays du sud-est de l'Asie induisent fréquemment des évaluations négatives à l'égard des produits industriels qui en proviennent, et peuvent en conséquence être jugés comme nonattractifs par les organisations qui rechercheraient un positionnement haut de gamme (Kotler et Gertner, 2002).

Il est connu que deux types d'organisations sont particulièrement à la recherche de légitimité (Abatecola, Cafferata et Poggesi, 2012) : les organisations jeunes, car elles ne sont pas 
connues, et celles de petite taille, car elles ne sont pas visibles. C'est ce qui explique le recours privilégié à cet usage des espaces géographiques de la part des start-up ou des jeunes créateurs de modes (Zimmerman et Zeitz, 2002).

\section{Discussion : les usages des espaces géographiques comme produits de conflits d'intérêts}

Si l'analyse proposée précédemment permet une meilleure compréhension du rôle des espaces géographiques dans la conduite des activités des organisations, elle nous amène aussi à souligner que les usages des espaces géographiques ne s'imposent pas d'euxmêmes. La définition de la nature des usages des espaces géographiques comme l'obtention du droit de faire usage de ces espaces font en effet fréquemment l'objet de luttes entre partiesprenantes (Briec, 2015).

D'une part, fait souvent négligé, la définition des usages organisationnels des espaces géographiques n'est pas une donnée : elle est le produit de conflits d'intérêts. Ainsi, en tant qu'exemple iconique, l'usage de la forêt Amazonienne est l'objet d'importants conflits entre les partisans d'un usage écologique, en vue d'une préservation radicale de la nature, les partisans d'un usage économique, en faveur de la sylviculture et de l'agro-industrie (usage qui est actuellement dominant), et les partisans d'un usage plus traditionnel par les amérindiens, davantage au carrefour d'usages écologiques, esthétiques et symboliques (Hecht, 2012). Afin de stabiliser une définition particulière d'un usage - organisationnel d'un espace géographique, des labels et des certifications sont régulièrement employés, avec plus ou moins de succès (pour un aperçu de l'échec de la certification forestière, voir Briec et Mandard, 2016). Par exemple, la création des parcs nationaux français vise spécifiquement à entériner la préservation d'espaces qui conjuguent propriétés écologiques et esthétiques, voire symboliques : «Un parc national peut être créé à partir d'espaces terrestres ou maritimes, lorsque le milieu naturel, particulièrement la faune, la flore, le sol, le sous-sol, l'atmosphère et les eaux, les paysages et, le cas échéant, le patrimoine culturel qu'ils comportent présentent un intérêt spécial et qu'il importe d'en assurer la protection en les préservant des dégradations et des atteintes susceptibles d'en altérer la diversité, la 
composition, l'aspect et l'évolution. » (Art. L331-1 du Code de l'environnement).

D'autre part, il peut également y avoir des conflits quant à l'obtention du droit de faire usage de ces espaces. À l'instar de la définition de l'usage, son appropriation peut en effet faire l'objet de luttes d'influence entre acteurs ou entre groupes d'intérêt, conflits qui peuvent prendre des formes extrêmes lorsque les espaces disponibles se font rares. Pour reprendre le cas emblématique de la forêt Amazonienne, des conflits particulièrement violents ont lieu pour l'appropriation des terres, en particulier entre les petits exploitants et les industriels de la sylviculture et de l'agriculture (Hecht, 2012).

Il est possible de noter que les conflits en matière de définition et d'appropriation surviennent fréquemment de manière concomitante. C'est ce à quoi l'on assiste dans le cas de la forêt Amazonienne. C'est aussi ce à quoi on assiste avec le projet Europacity de construction d'un gigantesque centre commercial dans le Val-d'Oise, qui voit s'affronter partisans de ce projet d'usage économique de l'espace et défenseurs des terres agricoles. Et c'est également ce que l'on observe en France dans le cadre des conflits portant sur les «zones à défendre», entre tenants de projets d'aménagement divers (e.g., l'aéroport Notre-Dame-des-Landes, le barrage de Sivens, le centre de stockage de déchets nucléaires de Bure) et opposants à ces projets, généralement favorables à un usage agricole des espaces géographiques faisant l'objet des conflits.

\section{Conclusion}

L'objectif de cet article était d'examiner un angle mort de la littérature managériale, bien souvent focalisée sur les problématiques de distance géographique ou d'internationalisation, en abordant la question des usages des espaces géographiques occupés par les organisations. Nous avons ainsi recensé ces différents usages, et montré que leur définition et leur appropriation pouvaient s'avérer conflictuels. Ce faisant, l'analyse que nous avons conduite offre un panorama d'un sujet particulièrement d'actualité, en témoignent les nombreux conflits en cours un peu partout en France et dans le monde. 


\section{Implications managériales}

Cette analyse nous permet tout d'abord de dégager une série d'implications pratiques.

Premièrement, en matière de stratégie d'entreprise, ce travail offre aux organisations une synthèse des options qui s'offrent à elles en matière d'usage des espaces géographiques, ce qui leur permet d'intégrer cette dimension dans leurs réflexions stratégiques. À une époque où la réputation constitue un actif clef pour nombre d'entreprises (Lange, Lee et Dai, 2011), il nous semble que les problématiques écologiques, esthétiques ou symboliques que nous évoquons ne peuvent plus être ignorées.

Deuxièmement, en matière de travail institutionnel, puisque les entreprises sont parties-prenantes dans la définition de l'usage des espaces géographiques puis dans leur usage effectif, cette analyse permet de souligner qu'il leur revient d'engager des démarches proactives en la matière. En particulier, les PME sont souvent moins investies sur ce type de problématique dont il faut qu'elles s'emparent (Lewis, Cassells et Roxas, 2015).

Finalement, troisièmement, en matière de responsabilité sociale des entreprises, ce travail permet de rappeler le fait que les entreprises peuvent être tenues pour responsable des usages qu'elles font des espaces géographiques dont elles disposent, et notamment de leurs conséquences environnementales. Les mesures légales vont d'ailleurs dans ce sens depuis quelques années, raison pour laquelle on voit se développer des pratiques de provisions comptables environnementales (Maurice, 2019).

\section{Implications scientifiques}

Cette analyse nous permet également de structurer les usages organisationnels des espaces géographiques en tant que champ de recherche. À la suite de quoi, nous proposons ici des pistes d'investigation futures.

Premièrement, la démarcation entre usage écologique et usage économique pourrait être clarifiée. Nous nous joignons ici à l'appel lancé par Whiteman et alii (Whiteman, Walker et Perego, 2013), afin d'investiguer davantage l'influence de l'activité des organisations sur les limites planétaires au travers d'une intégration 
des sciences naturelles à la littérature managériale. En particulier, s'il existe déjà toute une littérature sur le green supply chain management (Srivastava, 2007), celle-ci pourrait bénéficier d'une meilleure compréhension de ce que constituent des activités économiques environnementalement soutenables.

Deuxièmement, l'identification précise des causes et des conséquences de chacun des quatre usages pourrait être poursuivie. Par exemple, dans quelle mesure les organisations ont-elles recours à un usage symbolique des espaces géographiques? Dans quelle mesure l'esthétique d'un espace sur lequel une organisation est localisée estelle de nature à attirer les employés ou les clients ? Il pourrait être intéressant ici d'identifier les dynamiques qui s'instaurent en fonction des parties prenantes concernées.

Troisièmement, les possibilités de substitution ou de complémentarité entre les différents usages pourraient être examinés. Dans quelle mesure un usage écologique peut-il être substitué à un usage économique ? Dans quelle mesure les usages esthétiques et symboliques peuvent-ils être complémentaires ? Des problèmes de mesure ne manqueront pas de se poser ici, qui pourraient être résolus en recourant à une approche qualimétrique (Savall et Zardet, 2011).

Enfin, quatrièmement, les conflits quant à la définition de l'usage d'un espace géographique et quant à son appropriation subséquente pourraient eux aussi faire l'objet de davantage d'attention, et ce notamment dans le contexte actuel marqué par un essor des revendications environnementales. Historiquement élaborée dans le cadre d'un conflit quant à l'usage d'un espace géographique (voir Callon, 1986), la sociologie de la traduction pourrait être un cadre théorique tout à fait adapté à de telles investigations.

\section{Bibliographie}

Abatecola G, Cafferata R, et Poggesi S, «Arthur Stinchcombe's "liability of newness": contribution and impact of the construct ", Journal of Management History, vol. 18, n 4, 2012, p. 402-418.

Aggeri F, et Godard O, «Les entreprises et le développement durable », Entreprises et histoire, vol. , n 45, 2009, p. 6-19. 
Ambrose M. et Kulik C., «Old friends, new faces: Motivation research in the 1990s », Journal of management, vol. 25, n 3, 1999, p. 231-292.

Berchicci L, et King A, "Postcards from the edge: a review of the business and environment literature », The Academy of Management Annals, vol. 1, n 1, 2007, p. 513-547.

Boutaud A, et Gondran N, L'empreinte écologique, Paris: La Découverte, 2009.

Briec L, La place de l'environnement dans l'économie: une analyse régulationniste du cas du Brésil, Lille 1, 2015.

Briec L, et Mandard M, « L'échec de la certification forestière. Le cas de la certification FSC examiné au travers de la sociologie de la traduction ", Mondes en développement, vol. 1, n 173, p. 63-78, 2016.

Callon M. «Éléments pour une sociologie de la traduction. La domestication des coquilles Saint-Jacques dans la baie de SaintBrieuc », l’Année Sociologique, n³6, 1986, p. 169-208.

Cardinal J, «L'écolonomie au pays des Ch’tis. POCHECO: de l'enveloppe à l'écoveloppe ${ }^{\circledR}$ », Gestion, vol. 38, n 4, 2013, p. 90-95.

Chaumier C, « Un patrimoine de la communication d'entreprise. Les sièges sociaux parisiens des anciennes compagnies d'électricité », 1889-1946, Flux, vol. , n 4, 2010, p. 8-23.

DiMaggio P, et Powell W., « The iron cage revisited: Institutional isomorphism and collective rationality in organizational fields ", American sociological review, 1983, p. 147-160.

Gieryn T., "A Space for Place in Sociology », Annual Review of Sociology, vol. 26, 2000, p. 463-496.

Green J. et al., « Research priorities for managing the impacts and dependencies of business upon food, energy, water and the environment », Sustainability Science, vol. 12, n² 2, 2017, p. 319-331. 
Hecht S., «From eco-catastrophe to zero deforestation? Interdisciplinarities, politics, environmentalisms and reduced clearing in Amazonia ", Environmental conservation, vol. 39, $\mathrm{n}^{\circ}$ 01, 2012, p. 4-19.

Kirillova K, Fu X, Lehto X, et Cai L, «What makes a destination beautiful? Dimensions of tourist aesthetic judgment», Tourism Management, vol. 42, 2014, p. 282-293.

Kotler P, et Gertner D, « Country as brand, product, and beyond: A place marketing and brand management perspective », Journal of brand management, vol. 9, $\mathrm{n}^{\circ}$ 4, 2002, p. 249-261.

Krugman P., Geography and trade, MIT press, 1991.

Lange D, Lee P, et Dai Y, "Organizational reputation: A review », Journal of Management, vol. 37, $\mathrm{n}^{\circ} 1$, 2011, p. 153-184.

Lewis $\mathrm{K}$, Cassells $\mathrm{S}$, et Roxas $\mathrm{H}$, « SME and the potential for a collaborative path to environmental responsibility », Business Strategy and the Environment, vol. 24, n ${ }^{\circ}$, 2015, p. 750-764.

Loison M-C, et Pezet A, "L'entreprise verte et les boues rouges », Entreprises et histoire, vol. 4, n 45, 2006, p. 97-115.

Maurice J, «Quand les choix comptables liés à l'environnement ne sont pas qu'opportunistes: cas des provisions comptables environnementales », Finance Contrôle Stratégie, n²2-1, 2019.

Paquot T, Le paysage, La Découverte, 2016.

Pogutz S, et Martín-Tapia I, "Let the Environment Guide Our Development », Academy of Management Learning \& Education, p. amle.2017.0186, 2017.

Rockström J, et al., « A safe operating space for humanity », Nature, vol. 461, n 7263, 2009, p. 472-475. 
Ryan R, et Deci E «Intrinsic and extrinsic motivations: Classic definitions and new directions ", Contemporary educational psychology, vol. 25, $\mathrm{n}^{\circ} 1,2000$, p. 54-67.

Savall $\mathrm{H}$, et Zardet V, Recherche en sciences de gestion : Approche qualimétrique, Economica, 2004.

Srivastava S, "Green supply-chain management: A state-of-the-art literature review », International Journal of Management Reviews, Vol. 9, $\mathrm{n}^{\circ} 1,2007$, p. 53-80.

Weber M, Économie et société., 1921.

White $\mathrm{P}$, « Creative industries in a rural region: Creative West: The creative sector in the Western Region of Ireland », Creative Industries Journal, vol. 3, $\mathrm{n}^{\circ}$ 1, 2010, p. 79-88.

Whiteman G, Walker B, et Perego P, «Planetary boundaries: Ecological foundations for corporate sustainability », Journal of Management Studies, vol. 50, n² 2, 2013, p. 307-336.

Zimmerman M. et Zeitz G., «Beyond survival: Achieving new venture growth by building legitimacy ", Academy of Management Review, vol. 27, n³, 2002, p. 414-431.

Zimmermann J,-B, « Le territoire dans l'analyse économique », Revue française de gestion, vol. 4, n 184, 2008 p. 105-118. 